\title{
Joint-constraint model for large-eddy simulation of helical turbulence
}

\author{
Changping $\mathrm{Yu},{ }^{1,2}$ Zuoli Xiao, ${ }^{2,3, *}$ Yipeng Shi, ${ }^{2,3}$ and Shiyi Chen ${ }^{2,3}$ \\ ${ }^{1}$ LHD, Institute of Mechanics, Chinese Academy of Sciences, Beijing 100190, People's Republic of China \\ ${ }^{2}$ State Key Laboratory for Turbulence and Complex Systems, College of Engineering, Peking University, \\ Beijing 100871, People's Republic of China \\ ${ }^{3}$ Center for Applied Physics and Technology, College of Engineering, Peking University, Beijing 100871, People's Republic of China
}

(Received 10 February 2014; published 29 April 2014)

\begin{abstract}
A three-term mixed subgrid-scale (SGS) stress model is proposed for large-eddy simulation (LES) of helical turbulence. The new model includes a Smagorinsky-Lilly term, a velocity gradient term, and a symmetric vorticity gradient term. The model coefficients are determined by minimizing the mean square error between the realistic and modeled Leonard stresses under a joint constraint of kinetic energy and helicity fluxes. The model formulated as such is referred to as joint-constraint dynamic three-term model (JCD3TM). First, the new model is evaluated a priori using the direct numerical simulation (DNS) data of homogeneous isotropic turbulence with helical forcing. It is shown that the SGS dissipation fractions from all three terms in JCD3TM have the properties of length-scale invariance in inertial subrange. JCD3TM can predict the SGS stresses, energy flux, and helicity flux more accurately than the dynamic Smagorinsky model (DSM) and dynamic mixed helical model (DMHM) in both pointwise and statistical senses. Then, the performance of JCD3TM is tested a posteriori in LESs of both forced and freely decaying helical isotropic turbulence. It is found that JCD3TM possesses certain features of superiority over the other two models in predicting the energy spectrum, helicity spectrum, high-order statistics, etc. It is also noteworthy that JCD3TM is capable of simulating the evolutions of both energy and helicity spectra more precisely than other models in decaying helical turbulence. We claim that the present SGS model can capture the main helical features of turbulent motions and may serve as a useful tool for LES of helical turbulent flows.
\end{abstract}

DOI: 10.1103/PhysRevE.89.043021

PACS number(s): 47.27.E-

\section{INTRODUCTION}

The kinetic helicity is known as a global pseudoscalar, whose density is defined by the correlation between the velocity and vorticity $h=\boldsymbol{u} \cdot \boldsymbol{\omega}$, and can be either sign depending on the system of reference (left- or right-handed). Helical turbulence is characterized by the presence of nonzero mean helicity (or helicity integral), which is a topological invariant, measuring the knottedness, or amount of the linkage, of vortex lines [1]. The relative helicity $r_{h}=h /(|\boldsymbol{u}||\boldsymbol{\omega}|)$ represents a measure of the tendency of the velocity to align with the corresponding vorticity, and thus indicates the characteristics of local motions, i.e., helical $\left(r_{h} \rightarrow \pm 1\right)$ or nonhelical $\left(r_{h} \rightarrow 0\right)$ type. Helicity can lead to the formation of large-scale coherent structures in turbulent flows, though the underlying mechanisms still remain controversial [2-4]. Of particular importance are the roles played by helicity, for example, in the formation of tornadoes in atmospheric flows, the generation of magnetic fields in astrophysical flows [5], and the enhancement of mixing of chemical components [6].

The influence of helicity on the turbulence dynamics has been a matter of debate since the recognition that the kinetic helicity is a second inviscid integral invariant of threedimensional (3D) incompressible homogeneous and isotropic turbulence, in addition to the kinetic energy [1]. Two scenarios have been proposed for the cascade processes of the kinetic energy and helicity in helical turbulence [7]. One picture is that helicity cascades successively to smaller scales in the high-wave-number segment [with a helicity spectrum

*Corresponding author: z.xiao@pku.edu.cn
$H(k) \sim k^{-4 / 3}$ ], while energy is transferred to larger scales in the low-wave-number segment [with an energy spectrum $E(k) \sim k^{-7 / 3}$ ], analogous to the dual cascade of twodimensional (2D) turbulence [8-11]. This possibility was then excluded by Kraichnan [12] using a helical wave interaction theory and by André and Lesieur [13] through numerical simulation based on the so-called eddy-damped quasinormal Markovian (EDQNM) model. However, Kraichnan suggested in Ref. [12] that the helical motion be apt to slow down the cascade of energy to small scales, and, as a result, lead to the reduction of energy decay (a mechanism invoked to interpret the formation of long-lifetime strong storms [14]). This argument was further upheld by the works of Levich and Tsinober [15] as well as Pelz et al. [16], who pointed out that strong fluctuations of the local helicity might play an important part in the diminution of the energy cascade. This point of view originates from the inspection that intense helicity will result in severe reduction in the magnitude of the Lamb vector $\boldsymbol{\ell}=\boldsymbol{u} \times \boldsymbol{\omega}$, whose solenoidal part is responsible for the nonlinear interaction in Navier-Stokes turbulence. Nevertheless, Kraichnan and Panda [17] stressed that the alignment between the wave vector and the Lamb vector must be considered in order to understand the impact of helicity on the reduction of nonlinear transfer of energy. It was recently shown for freely decaying turbulence that, although the initial energy decay is suppressed in the presence of mean helicity, the self-similar decay rate is the same as in the nonhelical case [18]. Recently, Biferale et al. [19] found that the phenomenon of inverse energy cascade can possibly occur in all 3D turbulent flows if the mirror symmetry is broken by the single-signed helicity.

Another aspect is that both the energy and helicity are transferred from the driven scale to small scales (with their 
spectra following the Kolmogorov $-5 / 3$ law) until they are arrested by viscous dissipations. The joint or parallel cascade of the energy and helicity is supported by evidence from both direct numerical simulations [20-22] and experimental measurements of the atmospheric boundary layer flow [23]. It was shown through dimensional arguments and by using a shell model that there exists an inner scale $\xi$ for helicity dissipation, which is always greater than the Kolmogorov scale $\eta$ (characterizing the energy dissipation), and is increasingly so for helical turbulence with growing Reynolds number [24]. It was thus suggested that the helicity cascades linearly with the energy in an inertial range between the forcing scale and $\xi$, and the helicity dissipation dominates in another inertial range of energy between $\xi$ and $\eta$, where the turbulent motion is nonhelical. Recently, it was deduced by using a phenomenological approach that there should be a distortion time scale $\left(\tau_{H}\right)$ for the helicity cascade in analogy to that for the energy cascade $\left(\tau_{E}\right)$ [25]. The value of the ratio $\tau_{E} / \tau_{H}$ can be measured by the square root of the spectral relative helicity $(\alpha(k)=|H(k)| /[2 k E(k)] \sim 1 / k$ for the parallel cascade regime), and consequently, $\tau_{h}$ is comparable to $\tau_{E}$ even in the low relative helicity (or mean-helicity-free) limit. It was then demonstrated numerically that the joint cascade of energy and helicity is likely to be observed in two inertial subranges, i.e., a low-wave-number range with the spectra scaling as $k^{-5 / 3}$ and a higher-wave-number range scaling as $k^{-4 / 3}$. The former scaling law is determined by $\tau_{E}$, while the latter spectral dynamics is dominated by $\tau_{H}$. Besides, it was put forth that even the nonhelical homogeneous and isotropic turbulence possesses potential helicity from individual helical modes, which may have important effect on the spectral dynamics, especially in the predissipative range [26]. It was argued that the bulk helicity (or the total mean-flow helicity) plays a crucial part in the appearance of the axial-flow reversal in a turbulent swirling pipe flow [27].

Due to the overwhelming computational cost, direct numerical simulation (DNS) of the Navier-Stokes equations is still far from feasible for most engineering flows. The most striking technique besides DNS is large-eddy simulation (LES), which can ensure the fidelity of the flow fields to the largest extent, and has been widely used in engineering application and scientific research [28-30]. One of the the main tasks in LES approach is the modeling of the effects of subgrid-scale (SGS) motions based on the resolved quantities. A series of SGS models have been proposed for simulation of flows in various geometries, including the Smagorinsky model [31], the dynamic Smagorinsky model [32,33], the mixed model [34,35], the Lagrangian model [36], the scaledependent model [37-39], the vortex-based model [40,41], etc. It has been addressed that the incorporation of certain physical constraints in the SGS modeling is beneficial to avoiding unphysical solutions both in spectral space [42] and in physical space [43-45]. As manifested in Ref. [46], the variation process under an SGS dissipation constraint is helpful in improving the performance of a mixed dynamic model for simulation of driven and decaying isotropic turbulence. It was also shown that the inclusion of the near-wall Reynolds stress (and heat flux) constraint(s) in the SGS modeling allows the LES technique to simulate wall-bounded turbulent flows at moderate grid resolutions with promising accuracy $[47,48]$. Although the physical mechanism of the interplay between energy and helicity cascades still remains unclear, the effect of helicity has been taken into account for turbulence modeling. For example, Yokoi and Yoshizawa [49] studied the impact of helicity in inhomogeneous turbulence using a two-scale direct-interaction approximation (TSDIA) theory and proposed a three-equation $(k-\epsilon-H)$ model for a Reynolds-averaged Navier-Stokes (RANS) simulation of inhomogeneous turbulent flows. Efforts were also made to construct a second-order Reynolds stress model accounting for the effect of turbulent helicity on energy cascade [50]. Recently, improved spectral SGS model was developed based on the simultaneous evolution of the energy and helicity transfer terms in order to incorporate the effect of energyhelicity interactions [51,52]. The model with helical effect shows some advantages over that without helical effect in predicting the evolution of the spectra and velocity fields. In the light of the definition of the SGS helicity dissipation, $\mathrm{Li}$ et al. [53] suggested adding a vorticity gradient term to the traditional Smagorinsky formulation to yield a mixed helical model for LES of helical turbulence. Noticing that the vorticity gradient term is scale dependent even in the inertial subrange, $\mathrm{Yu}$ and Xiao [54] introduced a pseudo microscale (instead of the filter width) for the helical eddy diffusivity in the vorticity gradient term and proposed to calculate the model coefficients dynamically under a constraint of SGS helicity dissipation. The helical model refined as such improves the prediction of forced and decaying helical isotropic turbulence as compared with the traditional dynamic model in the absence of helicity flux constraint. Using a phenomenological analysis method, Yu et al. [55] derived a novel eddy-viscosity model, in which the eddy viscosity is closely associated with the correlation between the large-scale strain rate tensor and vorticity gradient tensor, a measurement of the resolved helicity dissipation rate.

To summarize, the turbulent helicity can have latent influence on the energy cascade process in both helical and non-helical turbulent flows. The presence of mean helicity may result in the formation of large-scale flow structures in6 the flow field. Therefore, the aim of this paper is to develop an SGS model for LES of helical turbulence, which is expected to have a high correlation with the real SGS stress and satisfy relevant physical constraints. Formally, a three-term dynamic SGS model is proposed, and the dynamic procedure is optimized by a joint constraint of SGS energy and helicity dissipations. The proposed model is tested a priori and a posteriori through simulation of forced and decaying helical isotropic turbulence. The calculated results are compared with those from DNS and LES using the unconstrained three-term model and other models.

\section{THEORETICAL ANALYSIS}

\section{A. Governing equations}

The basic equations for LES of incompressible turbulent flows are the coarse-grained (low-pass filtered) conservation equations of mass and momentum:

$$
\begin{gathered}
\partial_{i} \tilde{u}_{i}=0, \\
\partial_{t} \tilde{u}_{i}+\partial_{j}\left(\tilde{u}_{i} \tilde{u}_{j}\right)=-\frac{1}{\rho} \partial_{i} \tilde{p}+v \partial_{j} \partial_{j} \tilde{u}_{i}+\tilde{f}_{i}-\partial_{j} \tau_{i j} .
\end{gathered}
$$


Here, the tilde denotes spatial filtering at the grid scale $\Delta$, and $\widetilde{f}_{i}$ is the external driving force. $\tau_{i j}={\widetilde{u_{i}}}_{j}-\widetilde{u}_{i} \widetilde{u}_{j}$ is the SGS stress tensor, which needs to be modeled using the resolved velocity field $\tilde{u}_{i}$.

The resolved kinetic energy $\left(k_{\Delta}\right)$ is defined as $k_{\Delta}=\tilde{u}_{i} \tilde{u}_{i} / 2$. Thus, it is easy to derive from Eqs. (1) and (2) the governing equation for the resolved kinetic energy,

$$
\partial_{t} k_{\Delta}+\partial_{j}\left(\widetilde{u}_{j} k_{\Delta}\right)=\partial_{j} \widetilde{J}_{j}-\Pi_{\Delta}^{E}-2 \nu \widetilde{S}_{i j} \widetilde{S}_{i j}+\widetilde{f}_{i} \widetilde{u}_{i},
$$

where $\widetilde{S}_{i j}=\left(\partial_{j} \widetilde{u}_{i}+\partial_{i} \widetilde{u}_{j}\right) / 2$ is the resolved strain rate tensor, $\widetilde{J}_{j}=\tilde{u}_{i}\left(2 \nu \widetilde{S}_{i j}-\tilde{p} \delta_{i j} / \rho-\tau_{i j}\right)$ the spatial transport term of $k_{\Delta}$, and $\Pi_{\Delta}^{E}=-\widetilde{S}_{i j} \tau_{i j}$ the local SGS energy flux across scale $\Delta$. Here, $\delta_{i j}$ is the Kronecker delta.

Similarly, the resolved kinetic helicity is defined by $h_{\Delta}=$ $\tilde{u}_{i} \widetilde{\omega}_{i}$. One can show from the filtered Navier-Stokes equations [Eqs. (1) and (2)] and the large-scale vorticity $\left(\widetilde{\omega}_{i}\right)$ equation (not shown here) that the control equation for the resolved helicity reads

$$
\partial_{t} h_{\Delta}+\partial_{j}\left(\widetilde{u}_{j} h_{\Delta}\right)=\partial_{j} \widetilde{Q}_{j}-\Pi_{\Delta}^{H}-4 v \widetilde{S}_{i j} \widetilde{R}_{i j}+2 \widetilde{f}_{i} \widetilde{\omega}_{i},
$$

in which $\widetilde{R}_{i j}=\left(\partial_{j} \widetilde{\omega}_{i}+\partial_{i} \widetilde{\omega}_{j}\right) / 2$ is the resolved symmetric vorticity gradient tensor, $\widetilde{Q}_{j}=-2 \widetilde{\omega}_{i} \tau_{i j}-\varepsilon_{i j k} \widetilde{u}_{i} \partial_{l} \tau_{k l}-$ $\tilde{p} \widetilde{\omega}_{j} / \rho+\widetilde{u}_{i} \widetilde{u}_{i} \widetilde{\omega}_{j} / 2+2 v\left(\widetilde{u}_{i} \widetilde{R}_{i j}+\widetilde{\omega}_{i} \widetilde{S}_{i j}\right)-\varepsilon_{j k l} \widetilde{u}_{k} \widetilde{f}_{l}$ is the spatial transport term of $h_{\Delta}$, and $\Pi_{\Delta}^{H}=-2 \widetilde{R}_{i j} \tau_{i j}$ is the local SGS helicity flux across scale $\Delta$. Here, $\varepsilon_{i j k}$ is the alternating symbol.

\section{B. A three-term SGS model}

In order to account for the helicity effect and ensure the scale-invariant properties, Yu and Xiao [54] improved Li et al.'s [53] two-term SGS model by introducing a pseudoTaylor microscale $\left(\lambda_{\Delta}\right)$ to amend the helical term. The refined two-term helical model can be written in the form

$$
\tau_{i j}^{\text {mod }}=C_{1} \Delta^{2}|\widetilde{S}| \widetilde{S}_{i j}+C_{2} \lambda_{\Delta}^{2} \Delta|\widetilde{S}| \widetilde{R}_{i j},
$$

where $|\widetilde{S}|=\left(2 \widetilde{S}_{i j} \widetilde{S}_{i j}\right)^{1 / 2}$ is the magnitude of the resolved strain rate tensor, and $C_{1}$ and $C_{2}$ are the model coefficients to be determined. $\lambda_{\Delta}=\left(15\left\langle\widetilde{u_{i}} \cdot \widetilde{u_{i}}\right\rangle /\left\langle\widetilde{\omega}_{i} \cdot \widetilde{\omega_{i}}\right\rangle\right)^{1 / 2}$ scales as $\Delta^{1 / 2}$, and measures the ratio of the mean resolved energy to enstrophy at the grid scale $\Delta$. Here, $\langle\cdot\rangle$ represents an average over directions of statistical homogeneity or along pathlines.

The Smagorinsky-Lilly part in Eq. (5) is the simplest and most commonly used SGS model both for scientific research and engineering application. However, the traditional Smagorinsky-Lilly model has intrinsic drawbacks, such as being purely dissipative, being incapable of predicting the backscatter phenomenon, and having low correlation with the real SGS stress [33,40,56]. The second term in Eq. (5) may improve the performance of the mixed model, especially for the prediction of helicity cascade, as long as the model coefficient $C_{2}$ is determined in a proper way [54]. The gradient model (also referred to as nonlinear model), an approximation of the scale-similarity model, can give a better description of the SGS stress in comparison with the Smagorinsky-Lilly type model, and does not need a second filtering [57,58]. However, it is claimed that the SGS dissipation provided by the gradient model is insufficient [30]. Therefore, it was suggested that the gradient model be used in combination with the Smagorinsky model to form mixed model [34,35]. To take full advantage of the three models mentioned above, we suggest the following three-term SGS model

$$
\tau_{i j}^{\text {mod }}=C_{1} \Delta^{2}|\widetilde{S}| \tilde{S}_{i j}+C_{2} \Delta^{2} \frac{\partial \widetilde{u}_{i}}{\partial x_{k}} \frac{\partial \widetilde{u}_{i}}{\partial x_{k}}+C_{3} \lambda_{\Delta}^{2} \Delta|\widetilde{S}| \widetilde{R}_{i j} .
$$

The model coefficients $C_{1}, C_{2}$, and $C_{3}$ can be prescribed $a$ priori or calculated dynamically as the numerical simulation proceeds.

Dynamic SGS models were first investigated by Germano et al. [32], Lilly [33], and Meneveau et al. [36]. In these Smagorinsky-based dynamic models, the model coefficient is assumed to be scale invariant (within the inertial range) and is calculated simultaneously as the simulation progresses. Formally, a test filtering operation with a filter width $\alpha \Delta(1<$ $\alpha \leq 2$ ) is introduced to define the subtest-scale (STS) stress $T_{i j}={\widetilde{u_{i} u_{j}}}_{j}-\widetilde{\widetilde{u}}_{i} \widetilde{\widetilde{u}}_{j}$. Here, the overbar denotes spatial filtering at the test scale $\alpha \Delta$. The stresses at the grid and test scales are related to each other by the Germano identity [59],

$$
L_{i j} \equiv T_{i j}-\bar{\tau}_{i j}
$$

where $L_{i j}=\overline{\widetilde{u}}_{i} \widetilde{u}_{j}-\overline{\widetilde{u}}_{i} \overline{\widetilde{u}}_{j}$ is called resolved stress, which is a known quantity, given the resolved velocity field $\tilde{u}_{i}$. According to the proposed three-term SGS model [Eq. (6)], the corresponding formulation for the STS stress $T_{i j}$ reads

$$
\begin{aligned}
T_{i j}^{\mathrm{mod}}= & C_{1}(\alpha \Delta)^{2}|\overline{\widetilde{S}}| \widetilde{\widetilde{S}}_{i j}+C_{2}(\alpha \Delta)^{2} \frac{\partial \overline{\widetilde{u}}_{i}}{\partial x_{k}} \frac{\partial \widetilde{\widetilde{u}}_{j}}{\partial x_{k}} \\
& +C_{3} \lambda_{\alpha \Delta}^{2}(\alpha \Delta)|\overline{\widetilde{S}}| \widetilde{\widetilde{R}}_{i j} .
\end{aligned}
$$

Substituting $\tau_{i j}^{\text {mod }}$ and $T_{i j}^{\text {mod }}$ for $\tau_{i j}$ and $T_{i j}$ in Eq. (7) yields

$$
L_{i j}^{\mathrm{mod}}=T_{i j}^{\mathrm{mod}}-\bar{\tau}_{i j}^{\mathrm{mod}}
$$

The standard dynamic procedure for the three-term model [Eq. (6)] is to minimize the mean square error

$$
\mathcal{E}=\left\langle\left(L_{i j}-L_{i j}^{\mathrm{mod}}\right)^{2}\right\rangle
$$

with respect to the model coefficients $C_{1}, C_{2}$, and $C_{3}$, separately. The three-term SGS model with the model coefficients so determined is referred to as D3TM.

\section{Joint-constraint SGS model}

It has been demonstrated numerically that there exists a joint cascade of energy and helicity from the injection scale to smaller scales until they are arrested by the viscous dissipation [21,22]. Therefore, for high-Reynolds-number helical turbulence, the mean energy and helicity fluxes (or the so-called SGS dissipations) should remain nearly constant in the inertial subranges of energy and helicity (due to the negligibly small viscous effect), and approximately equal to the injection rates of energy and helicity, respectively. In physical space, the SGS dissipations of energy and helicity (due to the works done by the SGS stress against the large-scale strain and symmetric vorticity gradient) for given filter width $\delta$ are written as

$$
\varepsilon_{\delta}=\left\langle\Pi_{\delta}^{E}\right\rangle=-\left\langle\tau_{i j} \widetilde{S}_{i j}\right\rangle
$$

and

$$
\eta_{\delta}=\left\langle\Pi_{\delta}^{H}\right\rangle=-2\left\langle\tau_{i j} \widetilde{R}_{i j}\right\rangle .
$$


If the SGS dissipations [Eqs. (11) and (12)] are evaluated at the test scale $\alpha \Delta$, and the Germano identity [Eq. (7)] is employed, we can readily arrive at the following balance conditions for the SGS energy and helicity dissipations:

$$
\left\langle T_{i j} \widetilde{\widetilde{S}}_{i j}\right\rangle \equiv\left\langle\left(L_{i j}+\bar{\tau}_{i j}\right) \widetilde{\widetilde{S}}_{i j}\right\rangle
$$

and

$$
\left\langle T_{i j} \bar{R}_{i j}\right\rangle \equiv\left\langle\left(L_{i j}+\bar{\tau}_{i j}\right) \widetilde{\widetilde{R}}_{i j}\right\rangle .
$$

Replacing $T_{i j}$ and $\tau_{i j}$ with $T_{i j}^{\text {mod }}$ and $\tau_{i j}^{\text {mod }}$ in Eqs. (13) and (14) yields the following constraints on the SGS dissipations for any proposed SGS model:

$$
\left\langle T_{i j}^{\bmod } \widetilde{\widetilde{S}}_{i j}\right\rangle=\left\langle\left(L_{i j}+\bar{\tau}_{i j}^{\bmod }\right) \widetilde{\widetilde{S}}_{i j}\right\rangle
$$

and

$$
\left\langle T_{i j}^{\bmod } \widetilde{\widetilde{R}}_{i j}\right\rangle=\left\langle\left(L_{i j}+\bar{\tau}_{i j}^{\bmod }\right) \widetilde{\widetilde{R}}_{i j}\right\rangle .
$$

Shi et al. [46] applied the SGS energy dissipation constraint [Eq. (15)] to modeling of the SGS stress for LES of homogeneous isotropic turbulence, assuming that both grid and test scales are within the inertial range. Similarly, Yu and Xiao [54] utilized the SGS helicity dissipation constraint [Eq. (16)] to optimize a mixed SGS model for helical isotropic turbulence. In this paper, we propose to improve the performance of the dynamic three-term SGS model presented in Sec. II C by controlling both the mean energy flux and helicity flux using Eqs. (15) and (16). The model coefficients can be determined dynamically using the method of Lagrange multipliers as follows. Let $f_{k, i j}(k=1,2,3)$ represents the individual term in $\tau_{i j}^{\text {mod }}$, and $F_{k, i j}$ the corresponding term in $T_{i j}^{\text {mod }}$. Define $\alpha_{k, i j}=F_{k, i j}-\bar{f}_{k, i j}, \beta_{k}=\left\langle\left(F_{k, i j}-\bar{f}_{k, i j}\right) \overline{\widetilde{S}}_{i j}\right\rangle$, and $\gamma_{k}=\left\langle\left(F_{k, i j}-\bar{f}_{k, i j}\right) \widetilde{\widetilde{R}}_{i j}\right\rangle$. Thus, the joint-constraint dynamic three-term SGS model (JCD3TM) is implemented by minimizing the following error function with respect to the three model coefficients and the multipliers $\left(\lambda_{1}\right.$ and $\left.\lambda_{2}\right)$ :

$$
\begin{aligned}
\mathcal{E}^{L}= & \left\langle\left(L_{i j}-\sum_{k=1}^{3} C_{k} \alpha_{k, i j}\right)^{2}\right\rangle+\lambda_{1}\left(\sum_{k=1}^{3} C_{k} \beta_{k}-\Pi_{R}^{E}\right) \\
& +\lambda_{2}\left(\sum_{k=1}^{3} C_{k} \gamma_{k}-\Pi_{R}^{H}\right),
\end{aligned}
$$

where $\Pi_{R}^{E}=\left\langle\widetilde{\widetilde{S}}_{i j} L_{i j}\right\rangle$ and $\Pi_{R}^{H}=\left\langle\widetilde{\widetilde{R}}_{i j} L_{i j}\right\rangle$.

\section{NUMERICAL SIMULATIONS AND RESULTS}

\section{A. A priori tests}

In order to validate a priori the performance of the newly proposed models, we carried out DNS of helical 3D incompressible homogeneous isotropic turbulence by solving the forced Navier-Stokes equations using a pseudospectral solver in a cubic box with sides of length $2 \pi$. Periodic boundary conditions are imposed in all three directions. The Orszag 2/3 rule is used for dealiasing, and the second-order Adams-Bashforth scheme for the time integration. The system is driven by constant injection rates of energy and helicity, i.e., $\epsilon=0.1$ and $\eta=0.3$, respectively, in the first two wavenumber shells [53]. The latter can provide the system with a positive mean helicity. The initial condition is specified by a Gaussian random field with an energy spectrum of the form $E_{0}(k)=A k^{2} U_{0}^{2} k_{0}^{-5} e^{-2 k^{2} / k_{0}^{2}}$, with $k_{0}=4.5786$ and $U_{0}=0.715$. The grid resolution is $512^{3}$, and the kinematic viscosity $(v)$ is taken as $6 \times 10^{-4}$. The initial large-eddy turnover time is defined as $\tau_{0}=\pi /\left(k_{0} U_{0}\right)$. The system reaches a statistically steady state after about five large-eddy turnover times, and the resulting microscale Reynolds number $\left(R e_{\lambda}\right)$ is about 172. The Kolmogorov length scale, $\zeta \equiv\left(v^{3} / \epsilon\right)^{1 / 4}$, is 0.0068. A Gaussian filter is employed to implement the filtering operations. The performance of JCD3TM is compared with those of the traditional dynamic Smagorinsky model (DSM) and the dynamic mixed helical model (DMHM) [53].

As addressed in Sec. II C, the SGS dissipations of energy $\left(\varepsilon_{\delta}\right)$ and helicity $\left(\eta_{\delta}\right)$ are expected to remain constant if the filter width is within the inertial range of high-Reynolds-number helical turbulence. This argument has been numerically demonstrated both in spectral space [21] and in physical space [54]. One of the original goal of the proposed model (JCD3TM) is to predict the scale-invariant property of the SGS energy and helicity dissipations. Substituting $\tau_{i j}^{\text {mod }}$ in Eq. (6) for $\tau_{i j}$ in Eqs. (11) and (12) yields

$$
\begin{aligned}
& C_{1} f_{1}(\delta)+C_{2} f_{2}(\delta)+C_{3} f_{3}(\delta)=-1, \\
& C_{1} g_{1}(\delta)+C_{2} g_{2}(\delta)+C_{3} g_{3}(\delta)=-1 / 2,
\end{aligned}
$$

where $f_{1}(\delta)=\left\langle\delta^{2} \mid \widetilde{S}_{\mid} \widetilde{S}_{i j} \widetilde{S}_{i j}\right\rangle / \varepsilon_{\delta}, f_{2}(\delta)=\left\langle\delta^{2} \partial_{k} \widetilde{u}_{i} \partial_{k} \widetilde{u}_{j} \widetilde{S}_{i j}\right\rangle / \varepsilon_{\delta}$, $f_{3}(\delta)=\left\langle\lambda_{\delta}^{2} \delta|\widetilde{S}| \widetilde{R}_{i j} \widetilde{S}_{i j}\right\rangle / \varepsilon_{\delta}, g_{1}(\delta)=\left\langle\delta^{2}|\widetilde{S}| \widetilde{S}_{i j} \widetilde{R}_{i j}\right\rangle / \eta_{\delta}, g_{2}(\delta)=$ $\left\langle\delta^{2} \partial_{k} \widetilde{u}_{i} \partial_{k} \widetilde{u}_{j} \widetilde{R}_{i j}\right\rangle / \eta_{\delta}, g_{3}(\delta)=\left\langle\lambda_{\delta}^{2} \delta|\widetilde{S}| \widetilde{R}_{i j} \widetilde{R}_{i j}\right\rangle / \eta_{\delta}$. If the model coefficients $\left(C_{1}, C_{2}\right.$, and $\left.C_{3}\right)$ in D3TM and JCD3TM remain unchanged, a straightforward solution is that the nondimensionalized functions ( $f_{1}$ to $\left.g_{3}\right)$ maintain constant values for varying filter width $\delta$.

It has been shown that $f_{1}, f_{3}, g_{1}$, and $g_{3}$ are scale invariant in the inertial range of helical 3D incompressible homogeneous isotropic turbulence [54]. In this paper, the variation in the values of $f_{2}$ and $g_{2}$ with respect to the filter width $\delta$ is tested a priori using the DNS data for helical isotropic turbulence. We plot in Fig. 1 the dependence of $f_{2}(\delta)$ and $g_{2}(\delta)$ on the filter width $\delta$. It is seen that both $f_{2}(\delta)$ and $g_{2}(\delta)$ remain nearly constant when $\delta$ varies in the inertial range. Therefore, all three terms in JCD3TM [see Eq. (6)] satisfy the requirement of scale invariance.

Shown in Fig. 2 are the probability density functions (PDFs) of the modeled energy flux $\Pi_{\Delta}^{E}$ through the chosen filter scale $\Delta=80 \zeta$. The PDF for the real energy flux is also plotted for comparison. The horizontal axis is normalized by the energy input rate $\varepsilon$. All three models can predict the forward transfer of energy (all PDFs are positively skewed), but only JCD3TM can successfully capture the backscatters, which are believed to be a physical phenomenon in the 3D energy cascade [22]. The result for DMHM is similar to that for DSM because the contribution of the second term of DMHM is negligibly small in contrast with the first term when it predicts the physical quantities related to kinetic energy. Backscatters account for about $22 \%$ of the total space points in the field of real energy flux and about $15 \%$ in that of the modeled energy flux by JCD3TM. The modeled energy flux from JCD3TM correlates with the real energy flux at the level of $91 \%$, which is much higher than those of the other two models. 



FIG. 1. (Color online) Dependence of (a) $f_{2}(\delta)$ and (b) $g_{2}(\delta)$ on the filter width $\delta$ from an a priori test. The filter width is normalized by the Kolmogorov scale $\zeta$.

We display in Fig. 3 the PDFs of the modeled helicity flux $\Pi_{\Delta}^{H}$ across the given filter scale $\Delta=80 \zeta$. It can be seen that the PDF of the real helicity flux is nearly symmetric with small positive skew, which indicates transfer of positive helicity from large to small scales on average. DMHM and DSM are almost identical to each other, overestimating the PDF by an order of magnitude near the median $\Pi_{\Delta}^{H}$, and severely underestimating the probability and magnitude of tail events. The PDF of the modeled helicity flux from JCD3TM is much closer to that of the real flux, especially near the peaks. The local helicity fluxes

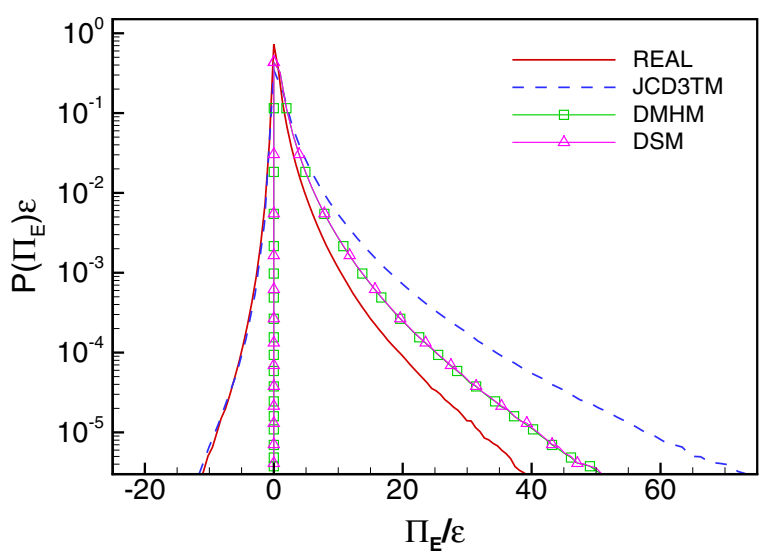

FIG. 2. (Color online) PDFs of the local energy flux $\Pi_{\Delta}^{E}$ for the filter width $\Delta=80 \zeta$ calculated using the JC3TDM (dashed line), DMHM (line with squares), DSM (line with triangles), and real SGS stress (bold solid line). The horizontal axis is normalized by the energy injection rate $\varepsilon$.



FIG. 3. (Color online) PDFs of the local helicity flux $\Pi_{\Delta}^{H}$ for the filter width $\Delta=80 \zeta$ calculated using the JCD3TM (dashed line), DMHM (line with squares), DSM (line with triangles), and real SGS stress (bold solid line). The horizontal axis is normalized by the helicity injection rate $\eta$.

predicted by JCD3TM, DSM, and DMHM correlate with the real helicity flux at levels of $93 \%, 13 \%$, and $14 \%$, respectively.

The pointwise correlation of the modeled SGS stress with the real stress can also measure the performance of the SGS models. We show in Fig. 4 the PDFs of one component of the modeled SGS stress $\left(\tau_{12}^{\bmod }\right)$ and the real SGS stress $\left(\tau_{12}\right)$ for the filter width $\Delta=80 \zeta$. It is observed that the PDFs for DMHM and DSM are much narrower than that for the real stress. The PDF of JCD3TM is close to that of the real stress. It is also noted that the PDFs of JCD3TM and the real stress are nearly symmetric, while those of DMHM and DSM are obviously skewed to the right. The pointwise correlation between JCD3TM and the real stress is as high as 94\%, while the correlations between the other two models and the real stress are much lower (less than 25\%). These observations also apply to other off-diagonal components of the SGS stress.

The probability of the new SGS model in simulating the local SGS energy and helicity fluxes can be seen more clearly in Figs. 5 and 6, which plot, respectively, the instantaneous

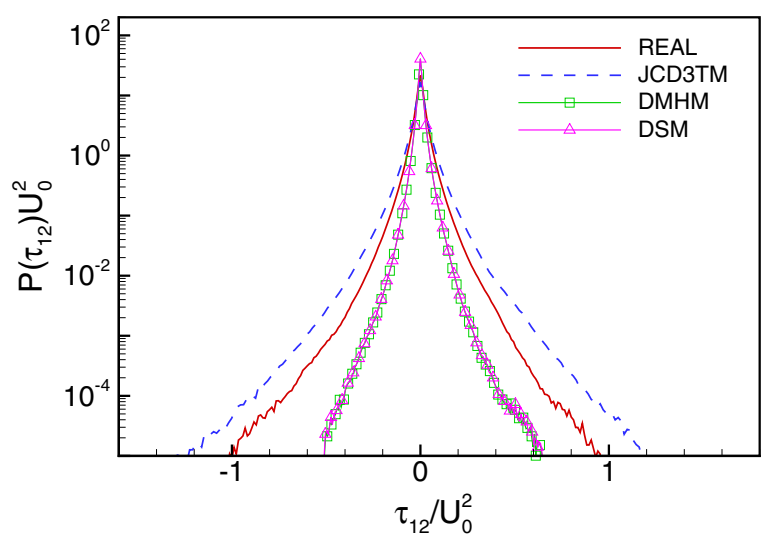

FIG. 4. (Color online) PDFs of the modeled SGS stresses $\tau_{12}^{\bmod }$ : JCD3TM (dashed line), DMHM (line with squares), DSM (line with triangles), and the real SGS stress $\tau_{12}$ (bold solid line) for the filter width $\Delta=80 \zeta$. The horizontal axis is normalized by the characteristic stress $U_{0}^{2}$. 

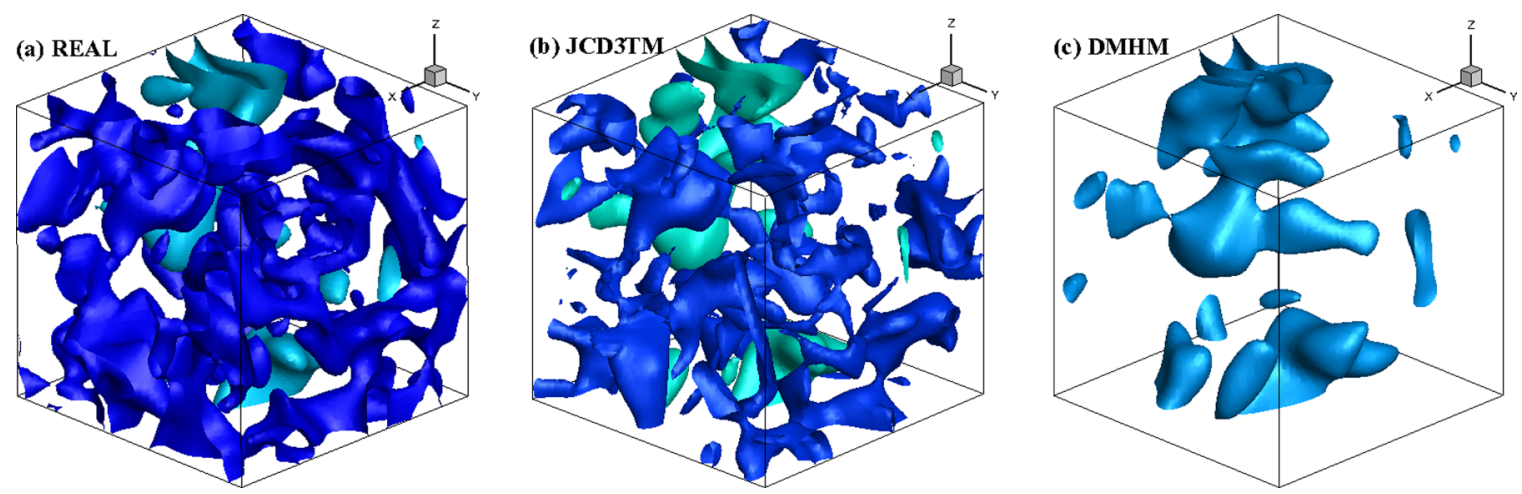

FIG. 5. (Color online) Instantaneous isosurfaces of the SGS energy fluxes $\Pi_{\Delta}^{E}$ calculated by (a) real SGS stress, (b) JCD3TM, and (c) DMHM for the filter width $\Delta=80 \zeta$.

isosurfaces of the energy flux $\left(\Pi_{\Delta}^{E}\right)$ and helicity flux $\left(\Pi_{\Delta}^{H}\right)$, calculated using the real SGS stress, JCD3TM, and DMHM at the filter scale $\Delta=80 \zeta$. The same levels of magnitude are shown for the three panels in each figure in a $128^{3}$ subdomain. Both the real energy and helicity fluxes are characterized by tubelike structures surrounded by sheetlike structures. To the eyes, JCD3TM can simulate the pointwise real fluxes more accurately than DMHM, especially for the energy flux. The behaviors of DSM are similar to those of DMHM, or even worse (not shown here).

Kraichnan [60] introduced a refined similarity hypothesis (RSH) for the local energy flux $\Pi_{\delta}^{E}$ to study the intermittency effects of the turbulent energy cascade. Kraichnan's RSH states that the structure functions of the velocity difference $\left\langle\left|\boldsymbol{u}\left(x_{i}+\boldsymbol{e}_{i} \delta, t\right)-\boldsymbol{u}\left(x_{i}, t\right)\right|^{p}\right\rangle$ and the structure functions of the local energy flux $\left\langle\left|\delta \Pi_{\delta}^{E}\right|^{p / 3}\right\rangle$ must have the same scaling exponents $\left(\zeta_{p}^{E}\right)$ with respect to the filter scale $\delta$. Here, $\boldsymbol{u}$ is the velocity vector field from DNS or experimental measurement, and $\boldsymbol{e}_{i}$ is the unit vector in the $i$ th direction. $\delta$ represents the filter width. Similarly, Chen et al. [22] suggested that the structure functions of the local helicity flux $\left\langle\left|\delta^{2} \Pi_{\delta}^{H}\right|^{p / 3}\right\rangle$ should have the corresponding intermittency exponent $\zeta_{p}^{H}$ for each $p$, and found that the helicity flux is more intermittent than the energy flux in helical 3D Navier-Stokes turbulence. In this paper, the capability of the new model in predicting such high-order statistics is also examined a priori. Shown in Fig. 7 are the $\frac{p}{3}$ th order structure functions of the normalized energy flux $\left\langle\left(\left|\delta \Pi_{\delta}^{E}\right| / u^{\prime 3}\right)^{p / 3}\right\rangle$ as function of the filter length scale $\delta$. Here, $u^{\prime}$ is the root-mean-square (r.m.s.) value of the DNS velocity fluctuations. The results obtained from real energy flux (solid line) are plotted to assess those predicted by different models (dashed lines). In order not to pollute the figures, only the results for $p=1,3,6,8$ are shown here. It is seen in Fig. 7(a) that JCD3TM can predict the energy flux structure functions very well when $p \leq 3$, especially in the inertial subrange $(65 \leq \delta / \zeta \leq 115)$, and behaves with the same tendency as the real SGS stress for $p>3$ despite the overestimated values of the structure functions. Both DMHM and DSM fail to predict the inertial-range structure functions of the energy flux for all p's [see Figs. 7(b) and 7(c)].

Similarly, we show in Fig. 8 the corresponding moments of the normalized helicity flux of different orders $\left\langle\left(\left|\delta^{2} \Pi_{\delta}^{H}\right| / u^{\prime 3}\right)^{p / 3}\right\rangle$ as functions of the filter scale $\delta$. It is obvious that JCD3TM can simulate the helicity flux structure functions accurately up to the third order, but tends to give much higher values of the higher-order $(p>3)$ structure functions. However, the curves given by DMHM and DSM deviate strongly from those by the real SGS stress, especially for large $p$ 's as can be seen in Figs. 8(b) and 8(c).

\section{B. A posteriori tests}

The proposed SGS model (JCD3TM) was then tested $a$ posteriori in LES of helical 3D incompressible homogeneous isotropic turbulence subject to external forcing. The results are compared with those from LESs using the other two models
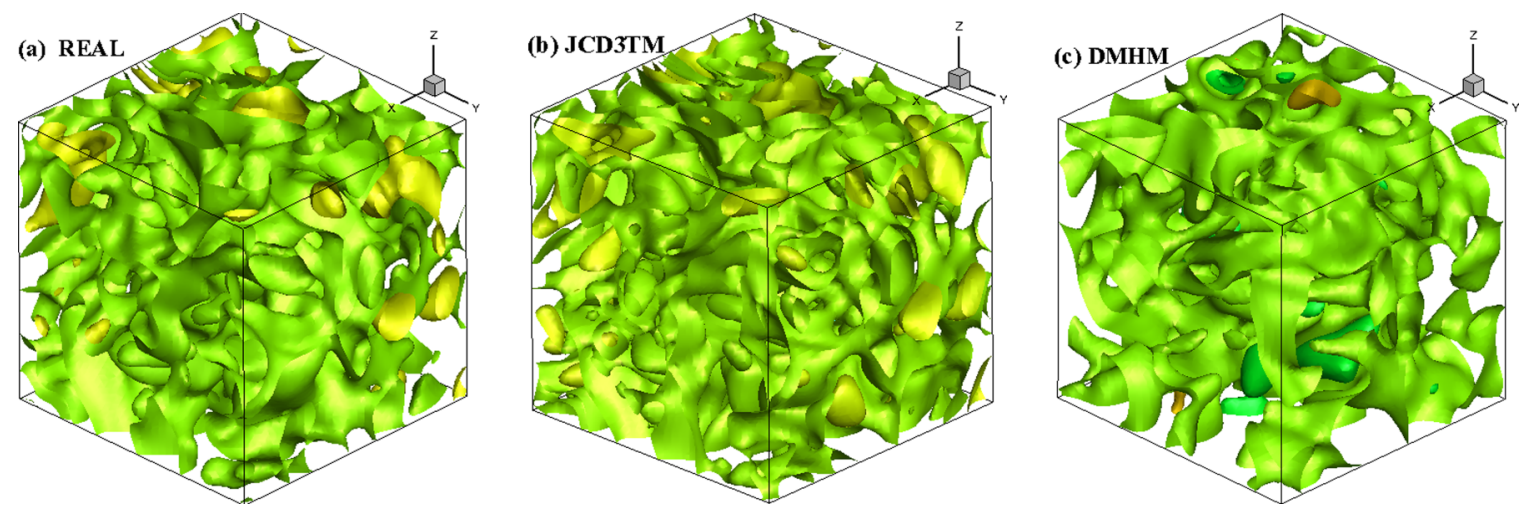

FIG. 6. (Color online) Instantaneous isosurfaces of the SGS helicity fluxes $\Pi_{\Delta}^{E}$ calculated by: (a) real SGS stress, (b) JCD3TM, and (c) DMHM for the filter width $\Delta=80 \zeta$. 



FIG. 7. (Color online) Moments of the normalized energy flux of order $p / 3$ as functions of the filter scale $\delta / \zeta$ : (a) JCD3TM, (b) DMHM, and (c) DSM. The model-predicted moments are denoted by dashed lines, and the real moments by solid lines. The inertial range of energy flux is located in the range $65 \leq \delta / \zeta \leq 115$. $u^{\prime}$ is the r.m.s. velocity.

and DNS. In LES, the filtered Navier-Stokes equations (1) and (2) are numerically solved with the SGS stress term $\tau_{i j}$ replaced by the models under consideration. The computational parameters are the same as those used in DNS mentioned in Sec. III B. The grid resolution is $64^{3}$.

In Fig. 9, we plot the energy spectra from LESs using various SGS models and the spectrum from DNS. Note that all the spectra shown here are obtained by averaging the simulated spectra over at least two large-eddy turnover times after the systems reach statistically steady state. It is seen that JCD3TM can accurately predict the energy spectrum for almost all wave numbers. DMHM and DSM, however, give similar spectral energy distribution, overestimating the energy
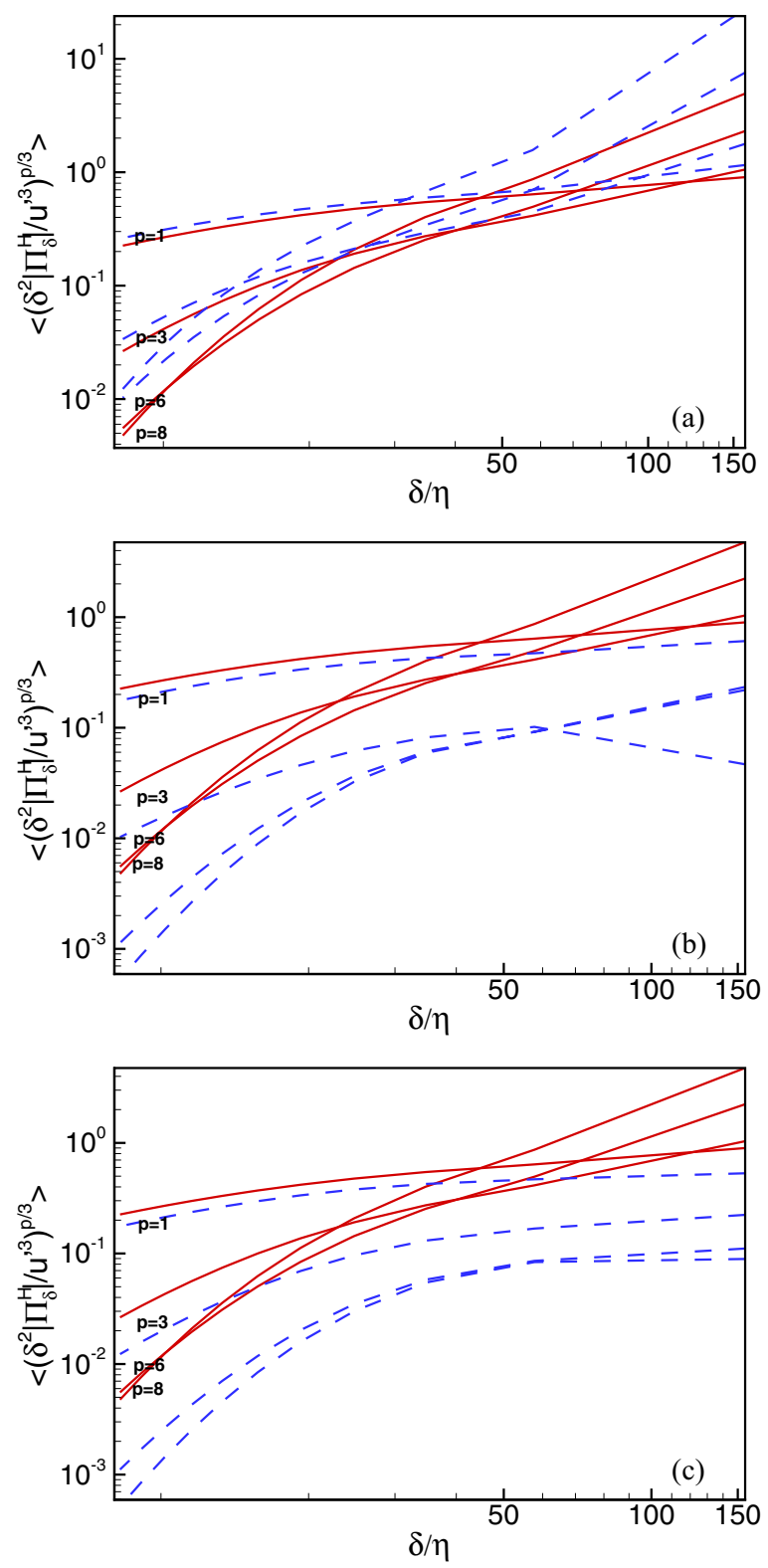

FIG. 8. (Color online) Moments of the normalized helicity flux of order $p / 3$ as function of the filter scale $\delta / \zeta$ : (a) JCD3TM, (b) DMHM, and (c) DSM. The model-predicted moments are denoted by dashed lines, and the real moments by solid lines. The inertial range of energy flux is located in the range $65 \leq \delta / \zeta \leq 115$. $u^{\prime}$ is the r.m.s. velocity.

spectrum in the low-wave-number range and underestimating the spectrum close to the grid scale. Therefore, the inclusion of the helical term in the dynamic two-term model makes subtle changes to the energy spectrum as compared with traditional DSM.

Shown in Fig. 10 are the helicity spectra from LESs using various SGS models in comparison with that from DNS. It is obvious that the spectrum predicted by JCD3TM coincides well with the DNS data tightly in most of the wave numbers. Within the low-wave-number range (i.e., $k \approx 3-9$ ), the helicity spectra given by DMHM and DSM are higher than that from DNS, and, near the grid scale, DMHM overestimates 




FIG. 9. (Color online) Energy spectra from LESs using different SGS models: JCD3TM (dashed line), DMHM (line with squares), and DSM (line with triangles). The energy spectrum from DNS (bold solid line) is plotted for comparison.

seriously the SGS dissipation, resulting in low spectral helicity distribution. Hence, DMHM is more dissipative than DSM in the case of a helical isotropic turbulence.

From Figs. 9 and 10, we acknowledge that the single Smagorinsky model tends to overrate the SGS energy dissipation, but underrate the SGS helicity dissipation, and that the two-term helical model would tend to overrate both the SGS energy dissipation and the SGS helicity dissipation. The introduction of the nonlinear term and the joint constraint of the SGS dissipation rates of energy and helicity remedies these deficiencies effectively.

Kurien et al. [25] suggested that helicity should have an effect on the long-term dynamics because the time scale for helicity transfer $\left(\tau_{H} \sim\left[\frac{1}{2}|H(k)| k^{2}\right]^{-1 / 2}\right)$ can remain comparable to the time scale for energy transfer $\left(\tau_{E} \sim\left[E(k) k^{3}\right]^{-1 / 2}\right)$ even for small eddies of size $\pi / k$. The ratio of $\tau_{E}$ to $\tau_{H}$ is measured by the square root of the spectral relative helicity $[|H(k)| / 2 k E(k)]^{1 / 2}$. In Fig. 11, we display the distribution of time-averaged spectral relative helicity $\langle H(k) / 2 k E(k)\rangle$ with respect to the wave number $k$ calculated using the LES and DNS data. For the joint cascade of energy and helicity, the

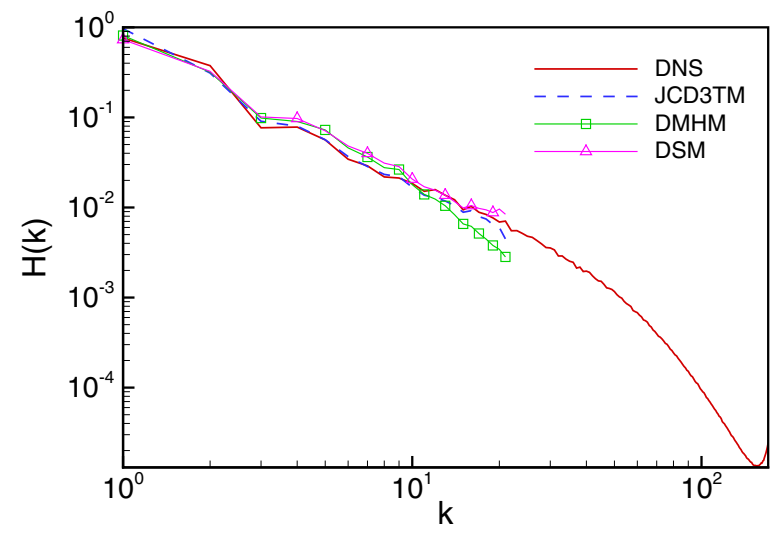

FIG. 10. (Color online) Helicity spectra from LESs using different SGS models: JCD3TM (dashed line) DMHM (line with squares), and DSM (line with triangles). The helicity spectrum from DNS (bold solid line) is shown for comparison.

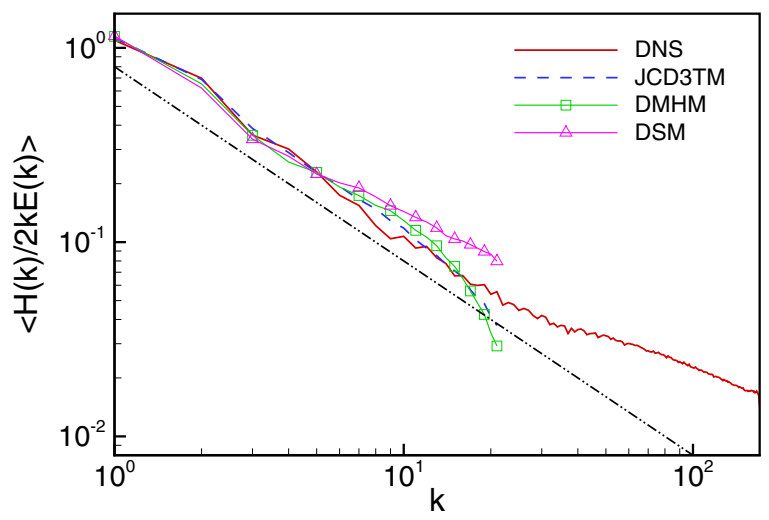

FIG. 11. (Color online) Distribution of time-averaged spectral relative helicity $\langle H(k) / 2 k E(k)\rangle$ as function of the wave number $k$ from LESs using different SGS models: JCD3TM (dashed line), DMHM (line with squares), and DSM (line with triangles). The result from DNS (bold solid line) is plotted for comparison. The dashed-double dotted line represents a power law $\left(\sim k^{-1}\right)$ scaling behavior.

spectral relative helicity is expected to scale as $k^{-1}$ in the inertial subrange $[7,25]$. It seems that DSM overestimates the relative helicity in the high-wave-number range, while both JCD3TM and DMHM underestimate it in the range close to the grid scale. The small-scale behavior of the relative helicity is highly associated with the spectral distributions of kinetic energy and helicity in the vicinity of the grid scale. However, the improvement in energy and helicity spectra does not necessarily imply the corresponding improvement in the spectral relative helicity, and vice versa. For example, both the energy and helicity spectra are underpredicted in the small scales by use of the DMHM. In the LES using the JCD3TM, the energy spectrum is identical to the DNS spectrum, while the helicity spectrum is a little bit underrated near the grid scale. Nevertheless, the relative helicity distributions given by these two models almost coincide with each other in the vicinity of the grid scale. It can also be seen that the result from JCD3TM displays a clear $k^{-1}$ scaling law range $(5<k<15)$ and is much closer to the DNS result in comparison with the other two models.

The most commonly invoked high-order statistics, which pertain to the turbulent motions of inertial-range scales, are the so-called longitudinal velocity structure functions $S_{n}^{L}(r) \equiv$ $\left\langle\left[u_{1}\left(x_{i}+\boldsymbol{e}_{1} r, t\right)-u_{1}\left(x_{i}, t\right)\right]^{n}\right\rangle$, where $r$ is the separation distance and $\boldsymbol{e}_{1}$ is the unit vector in the $x_{1}$ direction. In order to test the performance of the proposed and evaluated SGS models in predicting high-order statistics, the moments of longitudinal velocity difference for various separation distances $r$ are calculated using the LES data and filtered DNS data. Shown in Figs. 12(a), 12(b), and 12(c) are the longitudinal velocity structure functions of order 4,6 , and 8 , respectively, as functions of the separation distance. It is noted that, when $r>3 \Delta$, the curves predicted by JCD3TM almost coincide with the DNS data, while both DMHM and DSM show a tendency to underpredict slightly the DNS values, especially for large separation distances. When the separation distance approaches the grid scale, all three models fail to capture 

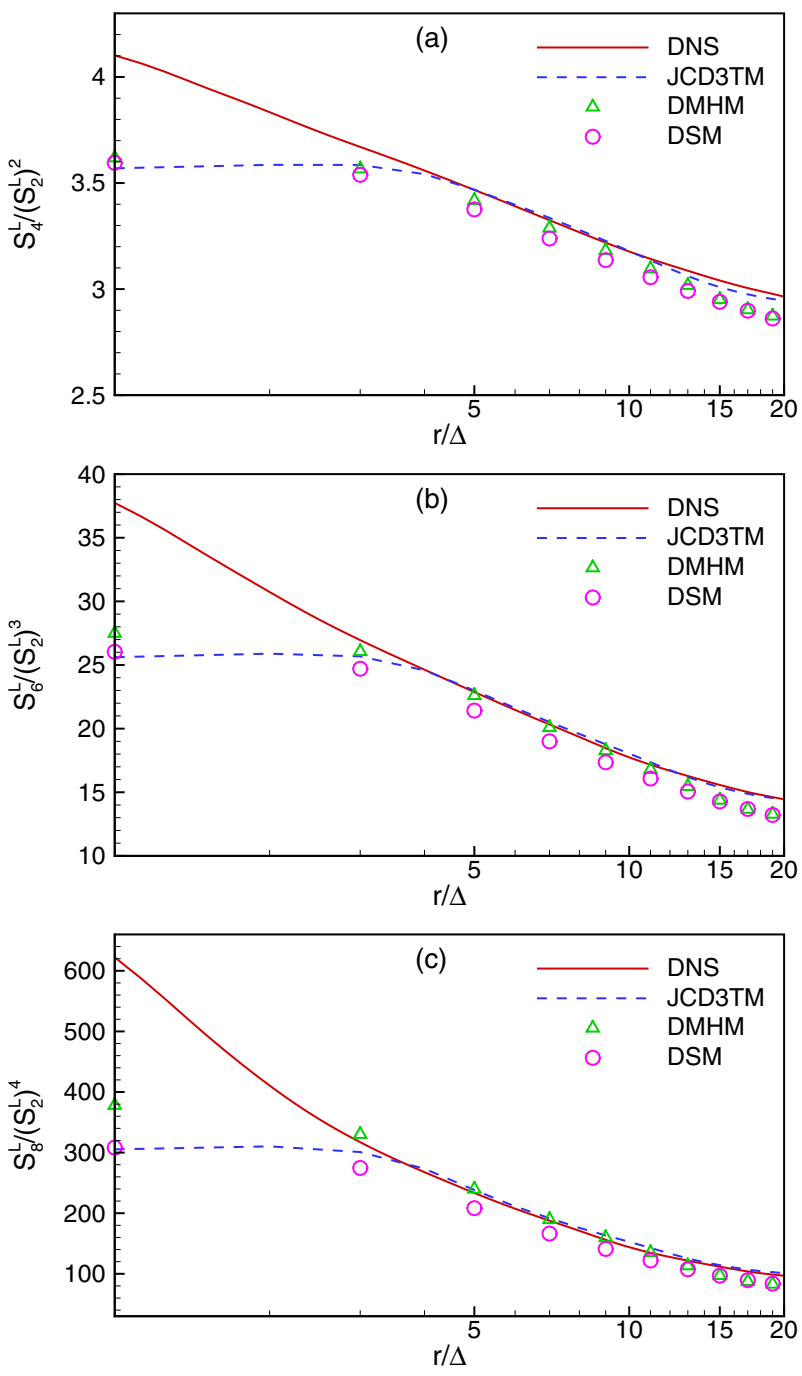

FIG. 12. (Color online) High-order moments of longitudinal velocity difference $\left(S_{n}^{L}\right)$ as functions of the separation distance $r$ : (a) $n=4$, (b) $n=6$, and (c) $n=8$. The horizontal axes are normalized by the LES grid scale $\Delta$, and the vertical axes by $\left(S_{2}^{L}\right)^{n / 2}$.

the high-order statistics, seriously underrating the structure functions in comparison with the DNS values.

High-order central moments provide us with statistical information on the spatial (or temporal) distribution of turbulent fluctuations about the mean value in the corresponding PDF. The most widely studied are the normalized moments of the longitudinal velocity derivative $M_{n}=$ $\left\langle\left(\partial u_{1} / \partial x_{1}\right)^{n}\right\rangle /\left\langle\left(\partial u_{1} / \partial x_{1}\right)^{2}\right\rangle^{n / 2}$. The skewness factor $\mathcal{S}=M_{3}$ measures the production rate of vorticity by the vortex- stretching process and the degree of irregularity of the turbulent fluctuations. The kurtosis (or flatness) factor $\mathcal{K}=M_{4}$ is another dimensionless metric for quantifying the degree of non-Gaussianity (or intermittency) of turbulence in the flow. For a symmetric Gaussian distribution, $\mathcal{S}=0$ and $\mathcal{K}=3$. A nonzero $\mathcal{S}$ and a value of $\mathcal{K}$ more than 3 are attributed to peaky signals associated with intermittent turbulent events. Homogeneous isotropic turbulence is characterized by a negative skewness factor $(\mathcal{S} \lesssim-0.3)$ and a positive flatness factor $(\mathcal{K} \gtrsim 4)$ [61]. Although Kolmogorov's hypotheses suggest that $\mathcal{S}$ and $\mathcal{K}$ are universal constants, numerical simulations and experimental measurements reveal that both $\mathcal{S}$ and $\mathcal{K}$ are Reynolds number dependent [61-63]. In order to assess the properties of the flow fields predicted by different SGS models, the skewness and flatness factors are calculated based on the velocity fields of driven helical isotropic turbulence obtained in LESs using JCD3TM, DMHM, and DSM. Listed in Table I are the skewness and flatness factors for different simulations. For comparison purpose, the results based on the DNS and filtered DNS fields are also presented. It is found that the skewness factor calculated based on the DNS data is about -0.53 , which is consistent with the result of Ishihara et al. [61] with $\mathcal{S} \sim-(0.32 \mp 0.02) \operatorname{Re}_{\lambda}^{0.11 \pm 0.01}$ at the present Reynolds number $\left(\operatorname{Re}_{\lambda}=172\right)$. Similarly, the flatness factor is about 6.33 and is also in good accordance with the fitted formula $\mathcal{K} \sim(1.14 \mp 0.19) \operatorname{Re}_{\lambda}^{0.34 \pm 0.03}$. All the models under consideration fail to capture these two high-order moments. This result is not surprising because the velocity-derivative moments pertain to the dissipative range of turbulence. The velocity derivative fields of LES are much less intermittent than those of DNS. This argument is supported by the corresponding skewness and flatness factors of the filtered DNS fields. It can also be seen that the JCD3TM is superior over the other two models in capturing the statistical property of the turbulent fluctuations, and the predicted values of $\mathcal{S}$ and $\mathcal{K}$ are closest to those of the filtered DNS fields.

The performance of the new SGS model is further evaluated in LES of freely decaying helical isotropic turbulence. For this purpose, the initial condition is obtained by truncating a statistically steady DNS velocity field (in Fourier space) at the LES grid scale, and the large-scale forcing term in Eq. (2) is turned off. The results for JCD3TM are compared with those from the other two models and DNS. We show in Figs. 13(a), 13(b), and 13(c) the energy spectra at three successive times $t=0,6 \tau_{0}$, and $12 \tau_{0}$ (with $\tau_{0}$ being the initial large-eddy turnover time) predicted by LESs using JCD3TM, DMHM, and DSM, respectively. The corresponding results from DNS are also plotted in each panel. As can be seen, JCD3TM predicts the evolution of energy spectra more accurately than DMHM and DSM. As in the case of

TABLE I. The skewness and flatness factors of the longitudinal velocity derivative obtained in DNS and LES by use of different SGS models.

\begin{tabular}{lrcrrr}
\hline \hline & \multicolumn{3}{c}{ Simulation method } \\
\cline { 2 - 5 } & DNS & Filtered DNS & LES-JCD3TM & LES-DMHM \\
\hline Skewness factor $\mathcal{S}$ & -0.53 & -0.44 & -0.36 & -0.31 & LES-DSM \\
Flatness factor $\mathcal{K}$ & 6.33 & 3.90 & 3.78 & 3.55 & 3.52 \\
\hline \hline
\end{tabular}



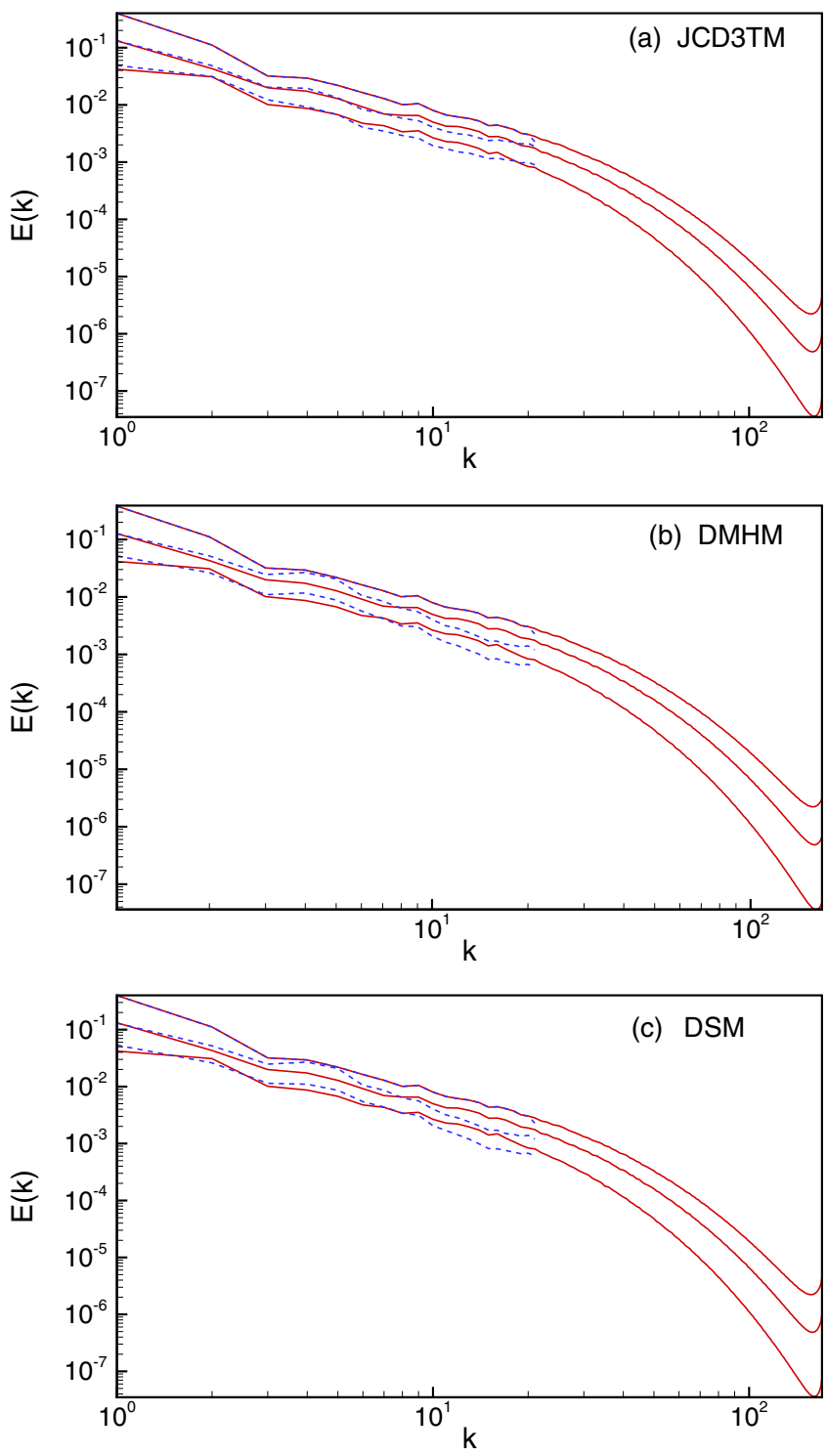

FIG. 13. (Color online) Evolution of energy spectra for freely decaying helical isotropic turbulence at three successive times $t=0$, $6 \tau_{0}$, and $12 \tau_{0}$ (dashed lines): (a) JCD3TM, (b) DMHM, and (c) DSM. The DNS results (solid lines) are also plotted as reference. $\tau_{0}$ is the initial large-eddy turnover time.

forced helical turbulence, DMHM and DSM are likely to overestimate the energy spectra in the low-wave-number range and underestimate them near the grid scale. Similarly, we display in Figs. 14(a), 14(b), and 14(c) the development of helicity spectra obtained in LESs utilizing the three SGS models. The DNS results are also presented for reference. The oscillations in the small-scale range of helicity spectra are caused by the pseudoscalar property of the local helicity. It is obvious that JCD3TM behaves much better than DMHM and DSM in predicting the helicity spectra as the turbulence decays progress. Therefore, it is inferred that the joint-constraint dynamic model can preserve its superiority in studying decaying helical turbulence as long as the scale-invariance of the models coefficients is approximately satisfied at the LES grid scale.
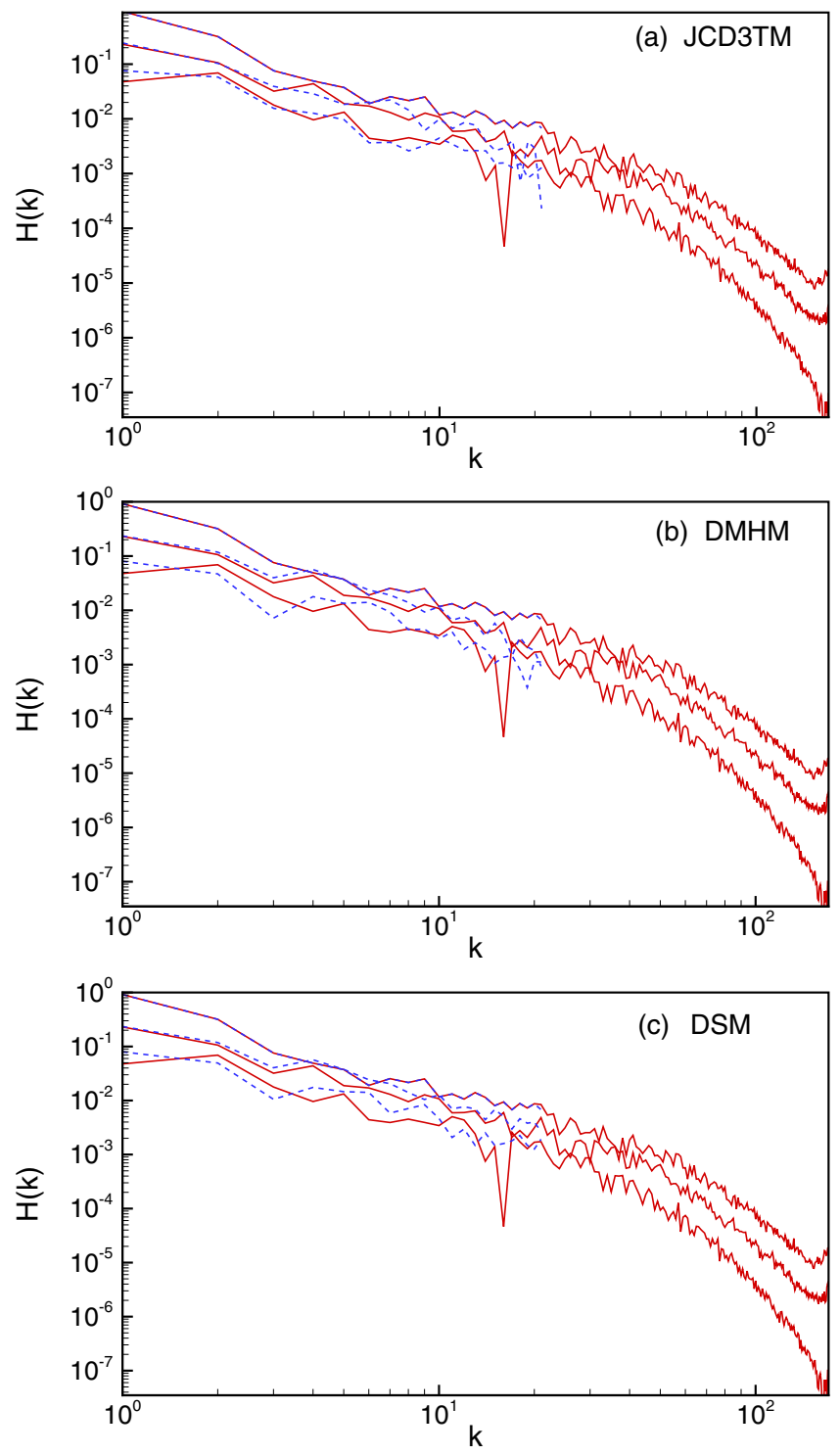

FIG. 14. (Color online) Evolution of helicity spectra for freely decaying helical isotropic turbulence at three successive times $t=0$, $6 \tau_{0}$, and $12 \tau_{0}$ (dashed lines): (a) JCD3TM, (b) DMHM, and (c) DSM. The DNS results (solid lines) are also plotted as reference. $\tau_{0}$ is the initial large-eddy turnover time.

\section{CONCLUSION AND DISCUSSION}

In this paper, a joint-constraint dynamic three-term model (JCD3TM) is presented for large-eddy simulation (LES) of helical turbulent flows. In the light of previously reported SGS models, the new model is designed to include a SmagorinskyLilly term, a nonlinear (velocity gradient) term, and a helical (symmetric vorticity gradient) term in order to incorporate the advantages from the three types of models, such as the properties of sufficient SGS dissipation and high correlation. Encouraged by the pioneer works of Shi et al. [46] and of $\mathrm{Yu}$ and Xiao [54], we introduce a joint constraint of the SGS energy and helicity fluxes to optimize the dynamic process for determining the model coefficients, according to the argument that helicity cascades linearly with energy in the inertial subrange of helical turbulence [21,22]. 
The inertial-range scale invariance of the model coefficients is validated a priori through investigating the fractional contribution of each term to the total SGS dissipations of energy and helicity. The performance of the proposed JCD3TM is tested both a priori and a posteriori in helical isotropic turbulence. The results are compared with those given by the dynamic mixed helical model (DMHM) introduced by Li et al. [53] and the traditional dynamic Smagorinsky model (DSM). In a priori tests, JCD3TM shows evident improvements in predicting probability density functions (PDFs) of the SGS stresses, energy and helicity fluxes, the spatial structures of energy and helicity fluxes, etc., in contrast with DMHM and DSM. In a posteriori tests, the LES using JCD3TM can predict the energy and helicity spectra more accurately than those using DMHM and DSM for both forced and freely decaying helical turbulence. JCD3TM also exhibits expected superiority over DMHM and DSM in calculating the high-order statistics of the two-point velocity difference, the energy and helicity fluxes, etc. It is noted that the dynamic three-term model (D3TM) without constraints achieves small improvement as compared with DMHM and DSM. The success of JCD3TM is ascribed to the comprehensive effects of the joint physical constraints and the proper combination of the celebrated model formulations. This work further manifests that the inclusion of physical constraint in the SGS modeling is of crucial importance for LES of turbulent flows.

It should be mentioned that the proposed model is still preliminary as it is, and the application of the model to numerical simulation of helical flows with complex geometry and engineering background is subject to further study. For example, the contribution of the nonlinear term in JCD3TM can be different when the proposed model is applied to simulating flows in different frames of reference. The present form of the model is not expected to perform better than other previously reported models when employed to simulate rotating turbulence. To account for the rotation effect induced by the Coriolis force, the velocity gradient tensor needs to be modified according to the angular velocity of the reference frame. Another issue may arise when the current model is applied to simulating turbulent flows at low Reynolds numbers or subject to wall boundary confinement, in which the scale-invariance assumption of the joint constraint (as well as the model coefficients) will usually be broken in the near-wall region or the whole flow domain. A scale-dependent (or a mesh Reynolds number-dependent) constraint for the SGS modeling needs to be raised in order for the present model to be able to mimic the low Reynolds number effect. This subject is worthy of further and more detailed research.

\section{ACKNOWLEDGMENTS}

We are grateful to Jianchun Wang and Kun Yang for many beneficial discussions on this work. Numerical simulations were carried out using the Dragon-1 cluster computer in the College of Engineering at Peking University and the Tianhe1A supercomputing facility at the National Supercomputer Center in Tianjin, China. We acknowledge the financial support provided by the National Natural Science Foundation of China (Grants No. 91130001, No. 11221061, No. 11372007 , and No. 11372330). This work was also supported by the 973 Program (Grant No. 2013CB834100) and the 863 Program (Grant No. 2012AA01A304).
[1] H. K. Moffatt, J. Fluid Mech. 35, 117 (1969).

[2] S. S. Moiseev, P. B. Rutkevich, A. V. Tur, and V. V. Yanovskiŭ, Sov. Phys. JETP 67, 294 (1988).

[3] O. A. Druzhinin and G. A. Khomenko, Advances in Turbulence 3, edited by A. V. Johansson and P. H. Alfredsson (SpringerVerlag, Berlin, 1991), pp. 151-154.

[4] G. V. Levina, S. S. Moiseev, and P. B. Rutkevich, in Advances in Fluid Mechanics, Nonlinear Instability, Chaos and Turbulence, Vol. 2, edited by L. Debnath and D. N. Riahi (WIT Press, Southampton, 2000), pp. 111-162.

[5] H. K. Moffatt and A. Tsinober, Annu. Rev. Fluid Mech. 24, 281 (1992).

[6] W. B. Zimmerman, in Fluid Mixing 5, IChemE Symposium Series 140 (Institute of Chemical Engineers, London, 1996), p. 213.

[7] A. Brissaud, U. Frisch, J. Léorat, M. Lesieur, and A. Mazure, Phys. Fluids 16, 1366 (1973).

[8] R. H. Kraichnan, Phys. Fluids 10, 1417 (1967).

[9] G. K. Batchelor, Phys. Fluids 12, II-233 (1969).

[10] S. Chen, R. E. Ecke, G. L. Eyink, X. Wang, and Z. Xiao, Phys. Rev. Lett. 91, 214501 (2003).

[11] Z. Xiao, M. Wan, S. Chen, and G. L. Eyink, J. Fluid Mech. 619, 1 (2009).

[12] R. H. Kraichnan, J. Fluid Mech. 59, 745 (1973).

[13] J. C. André and M. Lesieur, J. Fluid Mech. 81, 187 (1977).
[14] D. K. Lilly, J. Atmos. Sci. 43, 126 (1986).

[15] E. Levich and A. Tsinober, Phys. Lett. A 93, 293 (1983).

[16] R. B. Pelz, V. Yakhot, S. A. Orszag, L. Shtilman, and E. Levich, Phys. Rev. Lett. 54, 2505 (1985).

[17] R. H. Kraichnan and R. Panda, Phys. Fluids 31, 2395 (1988).

[18] T. Teitelbaum and P. D. Mininni, Phys. Rev. Lett. 103, 014501 (2009).

[19] L. Biferale, S. Musacchio, and F. Toschi, Phys. Rev. Lett. 108, 164501 (2012).

[20] V. Borue and S. A. Orszag, Phys. Rev. E 55, 7005 (1997).

[21] Q. Chen, S. Chen, and G. L. Eyink, Phys. Fluids 15, 361 (2003).

[22] Q. Chen, S. Chen, G. L. Eyink, and D. D. Holm, Phys. Rev. Lett. 90, 214503 (2003).

[23] B. M. Koprov, V. M. Koprov, V. M. Ponomarev, and O. G. Chkhetiani, Doklady Phys. 50, 419 (2005).

[24] P. D. Ditlevsen and P. Giuliani, Phys. Rev. E 63, 036304 (2001).

[25] S. Kurien, M. A. Taylor, and T. Matsumoto, Phys. Rev. E 69, 066313 (2004).

[26] E. Golbraikh, Int. J. Non-Linear. Mech. 44, 441 (2009).

[27] N. Yokoi and A. Yoshizawa, Phys. Fluids 16, 1186 (2004).

[28] U. Piomelli, Prog. Aerosp. Eng. 35, 335 (1999).

[29] M. Lesieur and O. Métais, Annu. Rev. Fluid Mech. 28, 45 (1996).

[30] C. Meneveau and J. Katz, Annu. Rev. Fluid Mech. 32, 1 (2000).

[31] J. Smagorinsky, Mon. Wea. Rev. 91, 99 (1963). 
[32] M. Germano, U. Piomelli, P. Moin, and W. H. Cabot, Phys. Fluids A 3, 1760 (1991).

[33] D. K. Lilly, Phys. Fluids A 4, 633 (1992).

[34] Y. Zang, R. L. Street, and J. R. Koseff, Phys. Fluids A 5, 3186 (1993).

[35] S. W. Liu, C. Meneveau, and J. Katz, J. Fluid Mech. 275, 83 (1994).

[36] C. Meneveau, T. S. Lund, and W. H. Cabot, J. Fluid Mech. 319, 353 (1996).

[37] C. Meneveau and T. S. Lund, Phys. Fluids 9, 3932 (1997).

[38] F. Porté-Agel, C. Meneveau, and M. B. Parlange, J. Fluid Mech. 415, 261 (2000).

[39] E. Bou-Zeid, C. Meneveau, and M. B. Parlange, Phys. Fluids 17, 025105 (2005).

[40] A. Misra and D. I. Pullin, Phys. Fluids 9, 2443 (1997).

[41] T. Voelkl, D. I. Pullin, and D. C. Chan, Phys. Fluids 12, 1810 (2000).

[42] R. H. Kraichnan and S. Chen, Physica D 37, 160 (1989).

[43] C. Meneveau, Phys. Fluids 6, 815 (1994).

[44] S. Ghosal, T. S. Lund, P. Moin, and K. Akselvoll, J. Fluid Mech. 286, 229 (1995).

[45] Y. Li and C. Meneveau, Phys. Fluids 16, 3483 (2004).

[46] Y. Shi, Z. Xiao, and S. Chen, Phys. Fluids 20, 011701 (2008).

[47] S. Chen, Z. Xia, S. Pei, J. Wang, Y. Yang, Z. Xiao, and Y. Shi, J. Fluid Mech. 703, 1 (2012).
[48] Z. Jiang, Z. Xiao, Y. Shi, and S. Chen, Phys. Fluids 25, 106102 (2013).

[49] N. Yokoi and A. Yoshizawa, Phys. Fluids A 5, 464 (1993).

[50] A. Yoshizawa, Phys. Rev. E 48, 273 (1993).

[51] J. Baerenzung, H. Politano, Y. Ponty, and A. Pouquet, Phys. Rev. E 77, 046303 (2008).

[52] J. Baerenzung, D. Rosenberg, P. D. Mininni, and A. Pouquet, J. Atmos. Sci. 68, 2757 (2011).

[53] Y. Li, C. Meneveau, S. Chen, and G. L. Eyink, Phys. Rev. E 74, 026310 (2006).

[54] C. Yu and Z. Xiao, Phys. Rev. E 87, 013006 (2013).

[55] C. Yu, R. Hong, Z. Xiao, and S. Chen, Phys. Fluids 25, 095101 (2013).

[56] K. Horiuti, Phys. Fluids A 1, 426 (1989).

[57] J. A. Langford and R. D. Moser, J. Fluid Mech. 398, 321 (1999).

[58] Y. Mornishi and O. V. Vasilyev, Phys. Fluids 13, 3400 (2001).

[59] M. Germano, J. Fluid Mech. 238, 325 (1992).

[60] R. H. Kraichnan, J. Fluid Mech. 62, 305 (1974).

[61] T. Ishihara, Y. Kaneda, M. Yokokawa, K. Itakura, and A. Uno, J. Fluid Mech. 592, 335 (2007).

[62] R. J. Hill, J. Fluid Mech. 452, 361 (2002).

[63] A. Gylfason, S. Ayyalasomayajula, and Z. Warhaft, J. Fluid Mech. 501, 213 (2004). 